\title{
Molecular Epidemiology and Genetic Diversity of Norovirus in Young Children in Phnom Penh, Cambodia
}

\author{
Kaewkanya Nakjarung, ${ }^{1}$ Ladaporn Bodhidatta, ${ }^{1}$ Pimmnapar Neesanant, ${ }^{1}$ \\ Paphavee Lertsethtakarn, ${ }^{1}$ Orntipa Sethabutr, ${ }^{1}$ Ket Vansith, ${ }^{2}$ \\ Chhour Y. Meng, ${ }^{2}$ Brett E. Swierczewski, ${ }^{1}$ and Carl J. Mason ${ }^{1}$ \\ ${ }^{1}$ Department of Enteric Diseases, Armed Forces Research Institute of Medical Sciences, 315/6 Rajvithi Road, \\ Bangkok 10400, Thailand \\ ${ }^{2}$ National Pediatric Hospital, 100 Russian Federation Boulevard, Phnom Penh, Cambodia
}

Correspondence should be addressed to Ladaporn Bodhidatta; ladapornb@afrims.org

Received 17 August 2016; Accepted 17 November 2016

Academic Editor: Jean-Paul J. Gonzalez

Copyright (C) 2016 Kaewkanya Nakjarung et al. This is an open access article distributed under the Creative Commons Attribution License, which permits unrestricted use, distribution, and reproduction in any medium, provided the original work is properly cited.

\begin{abstract}
This study investigated the genetic diversity of noroviruses identified from a previous surveillance study conducted at the National Pediatric Hospital in Phnom Penh, Cambodia, from 2004 to 2006. In the previous study, 926 stool samples were collected from children aged 3-60 months with acute diarrhea (cases) and without diarrhea (controls) with reported $6.7 \%$ of cases and 3.2\% of controls being positive for norovirus. The initial norovirus diagnostic assay was performed with real-time reverse transcriptionpolymerase chain reaction (real-time RT PCR) which also distinguished between genogroups I and II (GI and GII). Norovirus infection was most commonly detected in children aged 12-23 months in both cases and controls. Norovirus Genotyping Tool and phylogenetic analysis of partial sequences of the $3^{\prime}$ end of the RNA-dependent RNA Polymerase (RdRp) and the capsid domain region were employed to assign genotypes of the norovirus strains. GII.4 was the most predominant capsid genotype detected at $39.5 \%$ followed by GII.6 at 14.9\%. The GII.4 Hunter 2004 variant was the predominant strain detected. Six RdRP/capsid recombinants including GII.P7/GII.6, GII.P7/GII.14, GII.P7/GII.20, GII.P12/GII.13, GII.P17/GII.16, and GII.P21/GII.3 were also identified. This study of norovirus infection in young children in Cambodia suggests genetic diversity of norovirus as reported worldwide.
\end{abstract}

\section{Introduction}

Norovirus, a member of the family Caliciviridae, is an important human pathogen and is the leading cause of nonbacterial acute gastroenteritis outbreaks. Norovirus has been increasingly associated with sporadic episodes of acute gastroenteritis in children worldwide. It has been estimated that norovirus infections cause 1 million hospitalizations and 200,000 deaths in children under 5 years of age in the developing world [1].

The norovirus genome is organized into three open reading frames (ORF). ORF1 encodes six nonstructural proteins including the RNA-dependent RNA polymerase (RdRp); ORF2 encodes the capsid; and ORF3 encodes a small, minor structural protein [2]. Noroviruses are classified into at least 6 genogroups (GI-GVI) with a tentative genogroup VII based on the sequence diversity of the RdRP and capsid regions of the genome [3]. Genogroups I, II, and IV are known to infect humans. Genogroups are further subdivided into genotypes and there are 9 GI and 22 GII recognized genotypes based on the capsid sequence $[3,4]$. Despite an enormous genetic diversity, the majority of outbreaks and sporadic norovirus cases worldwide are associated with a single genotype from genogroup II, GII.4. Genotype GII.4 was responsible for $62 \%$ of reported norovirus outbreaks (4988) in 5 continents from January 2001 to March 2007 [5].

GII.4 variants have been reported as the major cause of norovirus gastroenteritis worldwide starting in 1995 with 
GII.4 variant Asia 2003 as the most widely circulated variant in Asia during 2003-2006 [6, 7]. In a Peruvian birth cohort study, $97 \%$ of characterized repeat norovirus infections were associated with a different genotype or a different GII.4 variant suggesting that genotype-specific immunity may develop with limited cross-protection within the genogroup which highlights the importance of identification and monitoring of GII.4 variants [8].

A potential mechanism that norovirus utilizes to evade host immunity is genetic recombination at the overlapping regions between the RdRp of ORF1 and the capsid protein encoding gene (ORF2), ORF1/ORF2 junction [9]. Multiple recombinants at this region have been reported such as GII.P4/GII.12 and GII.Pb/GII.3 in Japan [10] and GII.P9/GII.4 and GII.P9/GI.7 in Greece [11]. The variability of genetic recombination in norovirus suggests the need for a surveillance system to track the evolution of norovirus. An effective surveillance system would allow a better understanding of the burden of disease caused by norovirus and molecular epidemiology would also facilitate evolutionary analysis of norovirus.

There have been few reports on norovirus variants circulating in Southeast Asia [12-14] and how these norovirus variants compare to variants circulating elsewhere in the world. In the previous study of diarrhea etiology in young children in Phnom Penh, Cambodia, norovirus was the second most common virus detected following rotavirus [12]. In this study, norovirus positive samples from the previous study were further characterized and norovirus molecular epidemiology is reported including GII.4 variants and norovirus recombinants.

\section{Materials and Methods}

2.1. Study Design. A detailed description of the study design has been reported previously [12]. Briefly, children aged 3 months to 5 years were enrolled at the National Pediatric Hospital (NPH) in Phnom Penh between November 2004 and October 2006. Cases were enrolled among inpatient and outpatient children with acute diarrhea of no more than 72 hours' duration. Controls were children who visited the same hospital for other reasons and had not had diarrhea in the previous two weeks. Informed consent was obtained from one parent or a guardian for each participant. The study was approved by institutional review boards in both Cambodia and the United States.

2.2. Stool Collection and Nucleic Acid Extraction. Approximately $3-5 \mathrm{~g}$ of stool was collected from subjects. Stool samples were stored at $-70^{\circ} \mathrm{C}$ until processed. A $10 \%$ (wt/vol) stool suspension was prepared with distilled sterile water and total nucleic acids were extracted with NucliSens ${ }^{\circledR}$ Magnetic Extraction Kit (BioMerieux Inc., Durham, NC, USA) following the instructions of the manufacturer.

2.3. Real-Time Reverse Transcription (RT) PCR Screening and Genogrouping for Norovirus. The extracted nucleic acids were screened to identify the genogroup (GI and GII) by real-time RT PCR reactions as described previously [15]. The reactions were set up using the TaqMan ${ }^{\circledR}$ EZ RT PCR Core Reagent kit (Applied Biosystems, Foster City, CA, USA). All reactions were carried out in ABI PRISM 7900 Sequence Detector System and the results were analyzed with Sequence Detection Software version 2.1 (Applied Biosystems, Foster City, CA, USA).

2.4. Reverse Transcription (RT) PCR for the Cloning of ORF1/ORF2 Junction Regions. The extracted nucleic acids of norovirus positive samples were treated with DNase (Invitrogen, Carlsbad, CA, USA) to remove DNA prior to reverse transcription reaction. Five $\mu \mathrm{L}$ of RNA was reverse transcribed with primer G1SKR for GI and G2SKR for GII [16] to generate cDNA of the ORF1/ORF2 junction region using Multiscribe $^{\circledR}$ Reverse Transcriptase (Invitrogen, Carlsbad, CA, USA).

The ORF1/ORF2 junction was amplified using AmpliTaq Gold $^{\circledR}$ polymerase (Applied Biosystems, Foster City, CA, USA) containing a mixture of three forward primers (G1FF (A, B, and C) for GI or G2FB (A, B, and C) for GII) and reverse primer (G1SKR for GI or G2SKR for GII) [16, 17]. The thermocycling profile used was heat activation at $95^{\circ} \mathrm{C}$ for $10 \mathrm{~min}, 40$ cycles of denaturation at $95^{\circ} \mathrm{C}$ for $30 \mathrm{sec}$, annealing at $48^{\circ} \mathrm{C}$ for $30 \mathrm{sec}$, extension at $72^{\circ} \mathrm{C}$ for $1 \mathrm{~min}$, and postincubation at $72^{\circ} \mathrm{C}$ for $7 \mathrm{~min}$.

PCR products (GI $597 \mathrm{bp}$ and GII $468 \mathrm{bp}$ ) were cloned into a TA-Cloning vector (pCR 4.0-TOPO, Invitrogen, Carlsbad, CA, USA). One to three positive clones were sequenced from both forward and reverse directions using a commercial sequencing service (Macrogen, Seoul, Korea). DNA sequencing data were verified for consensus sequence using Sequencher software version 4.1.2 (Gene Codes Corporation, Ann Arbor, MI, USA).

2.5. Phylogenetic Analysis. The identification of norovirus genotype was performed by submitting sequences of the junction between RdRP and capsid genes to the online Norovirus Genotyping Tool (Version 1.0) [4]. Phylogenetic trees were also constructed based on sequences of RdRP and capsid genes. Nucleotide sequences of GI ( 448 bp correspond to nucleotides 4929 to 5376 of U07611 strain) and GII (436 bp corresponds to nucleotides 4929 to 5366 of U07611 strain) were aligned with representative reference strains (GI.Pa/GI.3-GQ856473, GI.Pc/GI.5-AB039774, GI.Pd/GI.3GQ856470, GI.P8/GI.8-GU299761, GII.P2/GII.2-X81879, GII.P3/GII.3-AB112332, JN176920, GII.P6/GII.6-JX989075, GII.P7/GII.7-AF414409, GII.P7/GII.6-AB504694, KM198549, GII.P7/GII.14-EF670650, GII.P7/GII.20-AB542917, GII.P12/ GII.12-AB525813, GII.P12/GII.13-AB354294, GII.P13/GII.13EU921354, GII.14-AY130761, GII.P16/GII.16-GQ856476, GII.P16/GII.17-AY502009, GII.P17/GII.17-KC597139, GII.P20/GII.20-EU275779, GII.P21/GII.21-EU019230, GII.P21/GII.3-KM198586, and GIII.1-EU360814 (out group)). For GII.4 variants determination, the nucleotide sequences from GenBank were used: GII.4 Yerseke 2006a-AB447433, GII.P12/GII.4 Asia 2003-AB220922, and GII.4 Hunter 2004-HM802542. Sequences of norovirus partial RdRp and capsids genes were submitted to GenBank. 
TABLE 1: Norovirus infection classified by genogroups in stool samples from children ( 3 months to 5 years) with acute diarrhea (cases) and nondiarrheal controls who attended the National Pediatric Hospital in Phnom Penh, Cambodia, between November 2004 and October 2006.

\begin{tabular}{lcc}
\hline & Cases $(N=580)$ & Controls $(N=346)$ \\
\hline Norovirus & $39(6.7 \%)^{* \#}$ & $11(3.2 \%)^{* \#}$ \\
GI & $4(0.7 \%)$ & $3(0.9 \%)$ \\
GII & $35(6.0 \%)^{*}$ & $8(2.3 \%)^{*}$ \\
\hline
\end{tabular}

${ }^{*} p<0.05$, chi-square test.

${ }^{*}$ Meng et al., 2011.

All sequences were aligned with ClustalW [18] in MEGA Version 6 [19]. Phylogenetic tree was constructed in MEGA Version 6 using neighbor-joining with Kimura twoparameter model with 1,000 bootstrap replicates.

2.6. Recombination Analysis. Simplot version 3.5 .1 was used to identify putative recombination breakpoints by comparing query sequence to nonrecombinant and recombinant parental strains [20]. Analysis parameters were as follows: Window: 200 bp, Step: 20 bp, GapStrip: On, Kimura (2parameter), and T/t: 2.0.

2.7. Statistical Analysis. The differences among proportions were analyzed by chi-square test and the difference between means was analyzed by $t$-test in IBM $^{\circledR}$ SPSS $^{\circledR}$ Statistics Version 22 (IBM Corp., Armonk, NY, USA).

\section{Results}

3.1. Norovirus Detection. A total of 926 stool samples ( 580 cases and 346 controls) were previously tested for the presence of norovirus and other enteric pathogens [12]. The prevalence of GI in cases and controls was $0.7 \%$ and $0.9 \%$ and the prevalence of GII was $6.0 \%$ and $2.3 \%$, respectively (Table 1) [12]. Children in the age group of 12-23 months had the highest prevalence in both cases and controls at $10.3 \%$ and $9.5 \%$, respectively (Figure 1). There is a significant difference in the mean ages of cases and controls with a mean age and standard deviation of $12.4 \pm 5.3$ months and $20.3 \pm 10.58$ months, respectively (Figure 1).

3.2. Sequence and Phylogenetic Analysis. Amplification of the ORF1/ORF2 junction region was successfully performed on 39 (7 cases and 32 controls) out of the 50 real-time PCR norovirus positive samples (2 GI and $37 \mathrm{GII}$ ). Repeated attempts to amplify the remaining 11 positive samples were unsuccessful. Identification of norovirus genotypes was achieved by cloning PCR products and performing sequence analysis on positive clones. The cloned PCR product corresponded to a 597 (GI) and a 468 (GII) bp fragment that maps to the overlapping region of ORF1 and ORF2.

A total of 43 nucleotide sequences were obtained. Four additional sequences were of mixed norovirus infection from the same sample. GenBank accession numbers of all sequences are KX685457-KX685499. These sequences were then submitted to the online Norovirus Genotyping Tool to

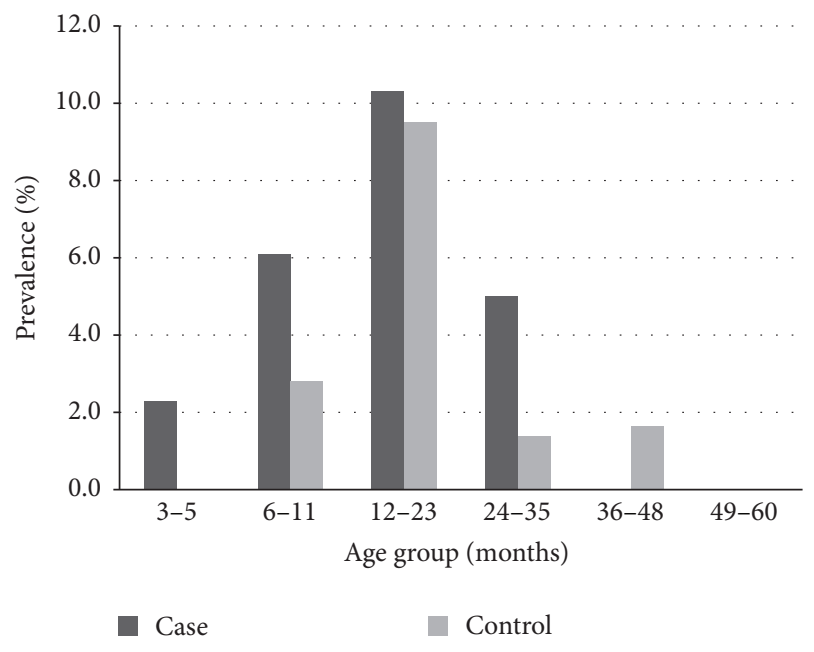

Figure 1: Prevalence of norovirus GI and GII infection in children with acute diarrhea (cases) and nondiarrheal controls by age. Samples were collected from children aged 3 months to 5 years who attended the National Pediatric Hospital in Phnom Penh, Cambodia, between November 2004 and October 2006. A significant difference ( $p<0.05, t$-test) in the mean of age of infection was observed between cases and controls averaging at 12.4 months and 20.3 months, respectively.

identify their genotypes based on partial RdRP and capsid genes. GII.4 was the most predominant capsid genotypes assigned at $39.5 \%$ followed by GII.6 at $14.9 \%$ (Table 2). GII.4 capsid variants that were identified included GII.4 Asia 2003, GII.4 Hunter 2004, and GII.4 Yerseke 2006a; however, 3 GII.4 sequences were not assigned any variant by the Norovirus Genotyping Tool. RdRP genotypes were assigned to $51.2 \%$ (22/43) of nucleotide sequences. Phylogenetic analysis of RdRP sequences clustered unassigned sequences into GII.P3 (1), GII.P4 (12), GII.P7 (5), GII.P12 (2), and GII.P21 (1) (Figures 2(a) and 2(b) and Table 2).

Two or more consensus genotype sequences were derived from 4 samples suggesting a mixed infection in these samples (Figures 2(a) and 2(b)). Mixed infections in this study are both inter- and intragenotype which included GII.P12/GII.12 with GII.P4/GII.4 Hunter 2004, GII.P6/GII.6 with GII.P4/GII.4 Yerseke 2006a, GII.P4/GII.4 Asia 2003 with GII.P7/GII.14, and GII.P4/GI.4 Hunter 2004 with GII.P4/GII.4 Asia 2003.

3.3. Recombination Analysis. Both of the Norovirus Genotyping Tool and phylogenetic analysis revealed 7 different recombinant genotypes from 16 sequences. GII.P7/GII.6 was the predominant recombinant with 6 sequences followed by GII.P7/GII.14 and GII.P7/GII.20 at 3 sequences each. Other recombinants were GI.Pc/GI.5, GII.P12/GII.13, GII.P16/GII.17, and GII.P21/GII.3 at 1 sequence each (Table 2). GII.P7 was the predominant RdRP that recombine with other capsid genotypes (GII.6, GII.14, and GII.20); however, there is no statistical significance between GII.P7 recombinants in case versus control samples in comparison to other recombinants identified in the study. 
TABLE 2: Summary of nonrecombinant (a) and recombinant (b) norovirus genotypes detected in case and control samples based on the Norovirus Genotyping Tool and phylogenetic analysis and Simplot for recombination analysis.

(a)

\begin{tabular}{|c|c|c|c|}
\hline \multicolumn{2}{|c|}{ Genotypes } & \multicolumn{2}{|c|}{ Number of samples } \\
\hline ORF1 & ORF2 & Case & Control \\
\hline GI.P8 & GI.8 & 1 & 0 \\
\hline GII.P2 & GII.2 & 1 & 0 \\
\hline GII.P3 & GII.3 & 2 & 0 \\
\hline GII.P4 & GII.4 Asia 2003 & 2 & 1 \\
\hline GII.P12 & GII.4 Asia 2003 & 3 & 1 \\
\hline GII.P4 & GII.4 Hunter 2004 & 5 & 2 \\
\hline GII.P4 & GII.4 Yerseke 2006a & 3 & 0 \\
\hline GII.P6 & GII.6 & 1 & 0 \\
\hline GII.P12 & GII.12 & 2 & 0 \\
\hline GII.P16 & GII.16 & 1 & 3 \\
\hline
\end{tabular}

Recombinants

\begin{tabular}{lcccccc}
\multicolumn{2}{c}{ Genotypes } & \multicolumn{2}{c}{ Number of samples } & Recombination position & Lordsdale nucleotide position & First report \\
ORF1 & ORF2 & Case & Control & - & - & Japan 1997-2001 [21] \\
\hline GI.Pc & GI.5 & 1 & 0 & - & $5022-5049$ & Burkina Faso 2011 [22] \\
GII.P7 & GII.6 & 5 & 1 & - & China [23 ${ }^{\#}$ \\
GII.P7 & GII.14 & 3 & 0 & - & $5022-5034$ & Brazil 2008 [24] \\
GII.P7 & GII.20 & 3 & 0 & $101-128$ & $5065-5073$ & South Korea 2004-2007 [25] \\
GII.P12 & GII.13 & 1 & 0 & $144-152$ & $5112-5122$ & South Africa 2010-2012 [26] \\
GII.P16 & GII.17 & 1 & 0 & $147-156$ & $5067-5076$ & Europe, Australia, Japan, 2002-2003 \\
GII.P21 & GII.3 & 1 & 0 & {$[10,27,28]$} & \\
\hline
\end{tabular}

Recombination breakpoint positions of each genotype and corresponding positions in the reference strain Lordsdale are reported.

* Numbering based on NP-492_2.

${ }^{\#}$ The original publication described the recombinant as GII.P6/GII.14 (GenBank accession number EF670650) but the Norovirus Genotyping Tool and phylogenetic analysis showed that it was closely related to GII.P7/GII.14.

All of the 16 recombinant sequences were subjected to Simplot analysis to determine recombination breakpoints. Table 2 shows a range of nucleotide breakpoint of each recombinant in comparison to the reference strain Lordsdale, accession number X86557, which falls into ORF1/ORF junction. Representatives of Simplot of each recombinant are shown in Figure 3.

\section{Discussion}

The presence of norovirus in cases of pediatric diarrhea in Cambodia was described previously but little is known about the genetic diversity of the circulating norovirus strains $[12,29]$. In this study, the percentage of norovirus positive cases among children with diarrhea seen at the hospital $(6.7 \%)$ is relatively low compared to studies from neighboring Southeast Asian countries [13, 14, 30]. This does not necessarily reflect the true burden of norovirus gastroenteritis in Cambodia for several reasons. One of the possible limiting factors was that it was a passive surveillance where sample collection was performed at a single hospital where possible sample bias can be introduced. Additionally, the low percentage is perhaps from underreported norovirus diarrhea cases to the tertiary care hospital as norovirus associated diarrhea may not be severe or it is an uncommon practice to seek healthcare for diarrheal disease in Cambodia. The finding from a community-based surveillance study in Cambodia reported that, even among severe cases of diarrhea, less than $30 \%$ sought treatment from a healthcare facility [31]. Additionally, it may be possible that norovirus is overshadowed by the presence of other pathogens in low-income settings where sanitary measures are limited as evidenced by higher percentages of bacteria and rotavirus detected in the previous report $[12,32]$. Norovirus prevalence has become more prominent in higher-income settings where other pathogens are controlled through improvement of public health control measures for water and sanitation [32]. Additional systematic surveillance will be required to fully understand the burden of norovirus infection in Cambodia.

Age distribution of norovirus infection in this study was similar to what has been reported elsewhere [33-35]. Children in the 3-5-month age group were not commonly infected with norovirus, possibly due to maternal immunity and the protective benefit of breast-feeding [36]. The prevalence was highest in both cases and controls in the 12-23-month age range and declined after 24 months of 


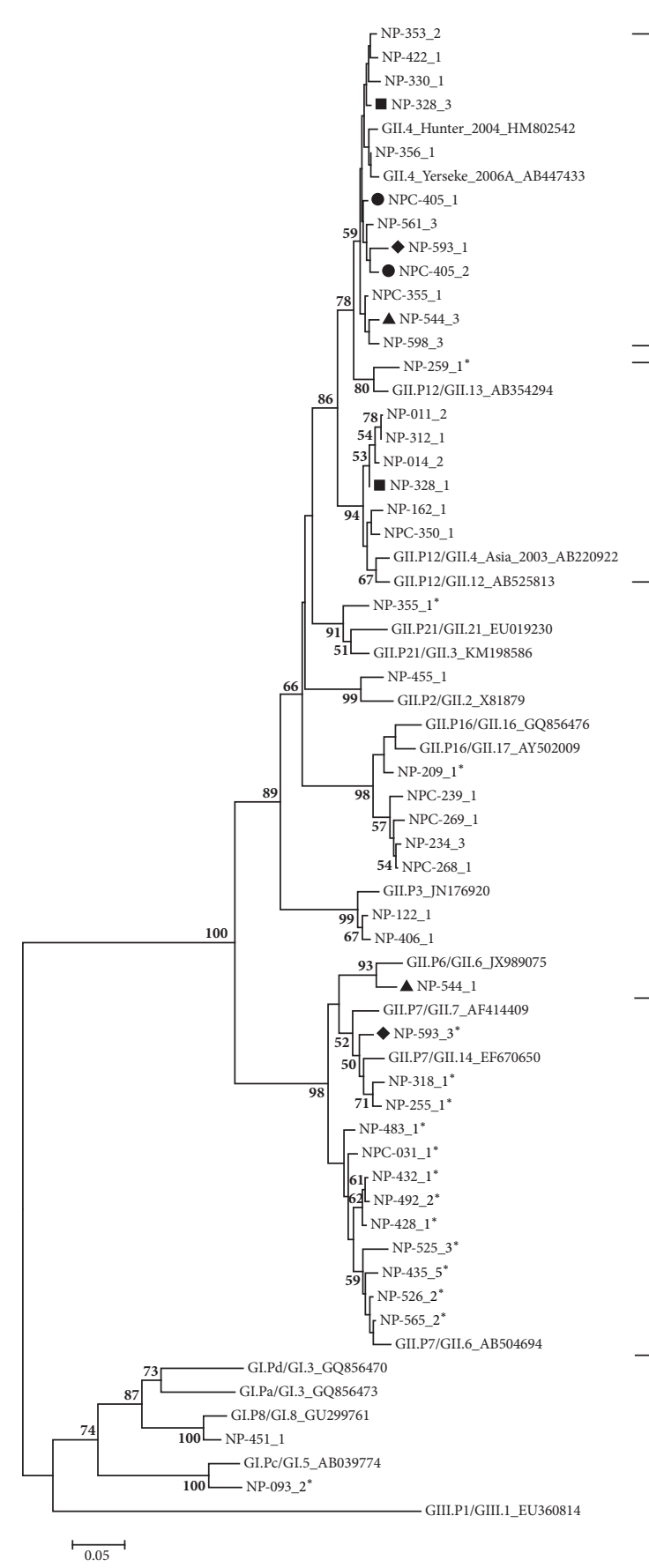

(a)

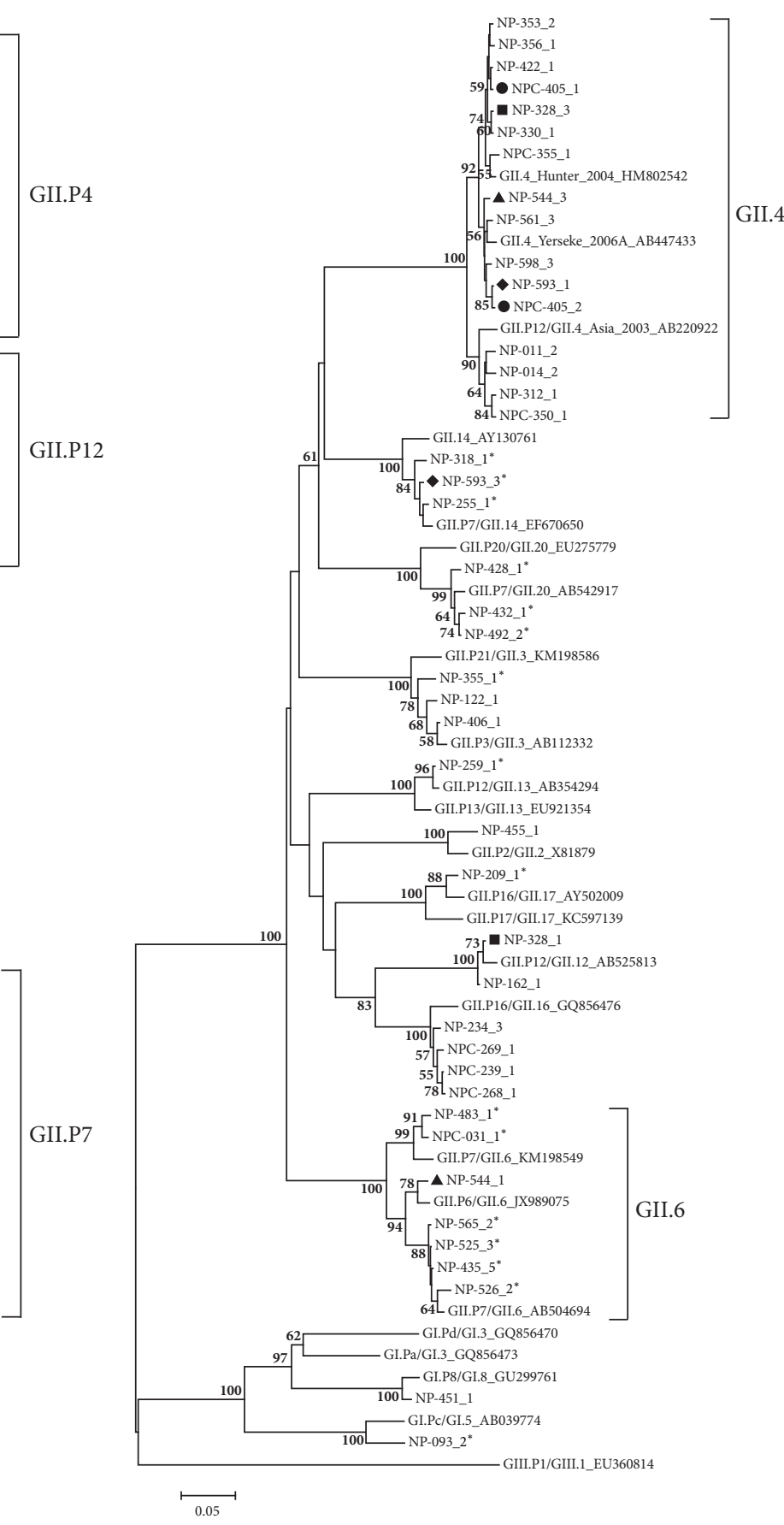

(b)

FIGURE 2: Phylogenic analysis of partial RNA-dependent RNA polymerase (RdRP) (a) and capsid genes (b) of norovirus from Cambodia pediatric samples during 2004-2006. Sample names are indicated as NP \#\#-\# for case and NPC \#\#-\# for control. -\# after the sample name indicates colony number of clone for that sample. Recombination strains are indicated by ${ }^{*}$ at the end of sample name. Mixed infections from same sample are indicated with similar symbol ( $\square$ for NP-328, $\bullet$ for NPC-405, $\boldsymbol{\Delta}$ for NP-544, and $\uparrow$ for NP-593).

age. Information on age distribution of norovirus infection is important for targeting population for norovirus vaccine implementation.

Studies of sequence and genotypic analysis of norovirus positive samples described GII.4 as the most common genotype detected in both outbreaks and sporadic cases of acute gastroenteritis worldwide [5]. The 16 isolates of GII.4 identified in this study were classified into 3 variants: Hunter 2004, Yerseke 2006a, and Asia 2003, corresponding to reported norovirus genotypes circulating during 2004-2006 


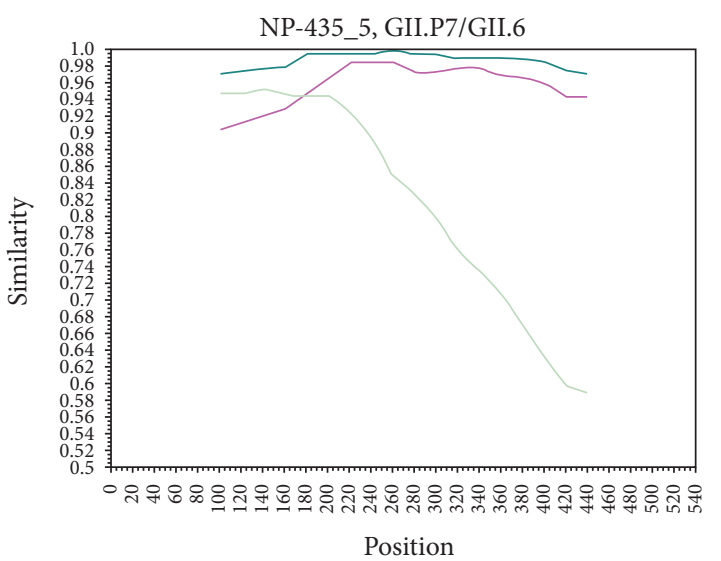

— GII.P6/GII.6_AB039779_ — GII.P7/GII.6_AB504694 GII.P7/GII.7_AF5414409

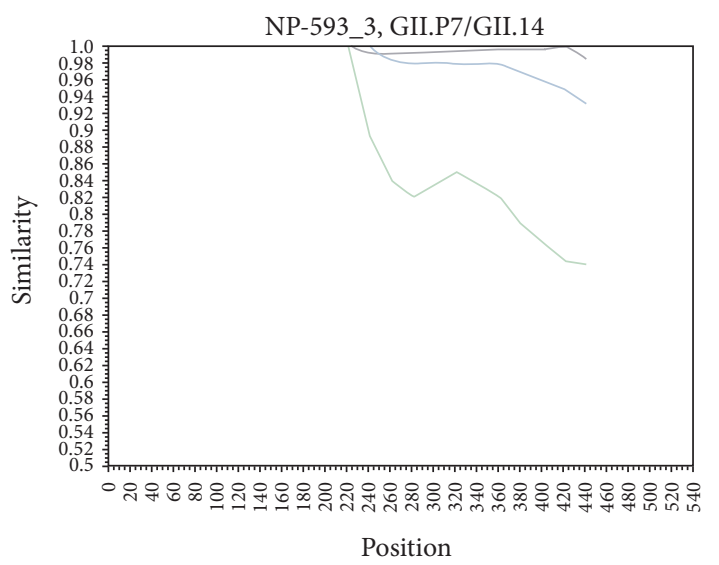

GII.P7/GII.7_AF414409 - GII.P7/GII.14_EF670650 — GII.14_AY130761

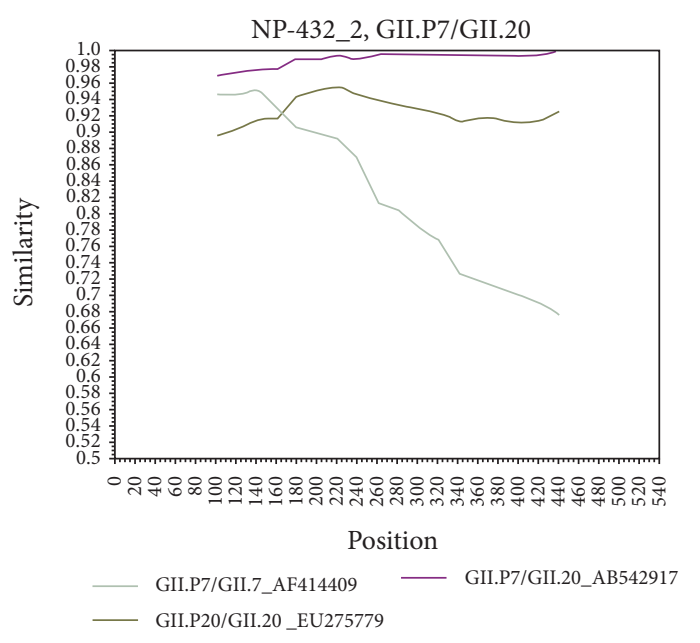

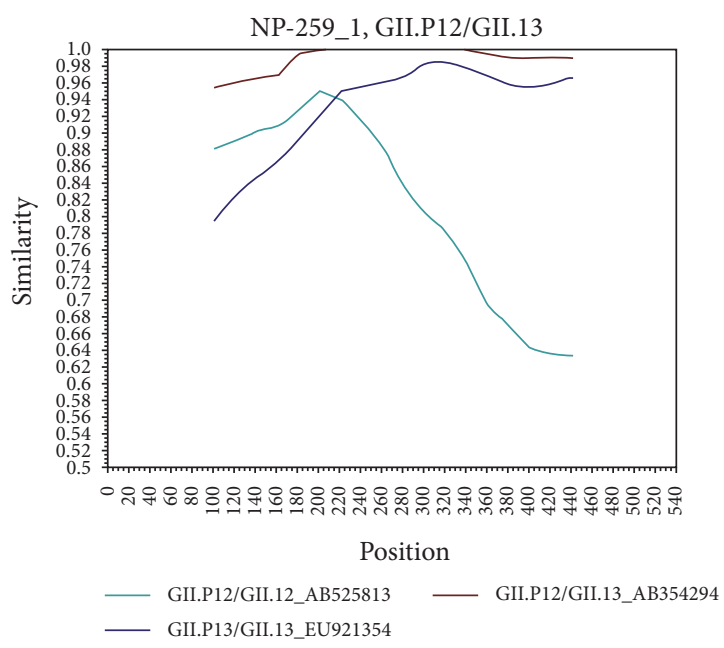

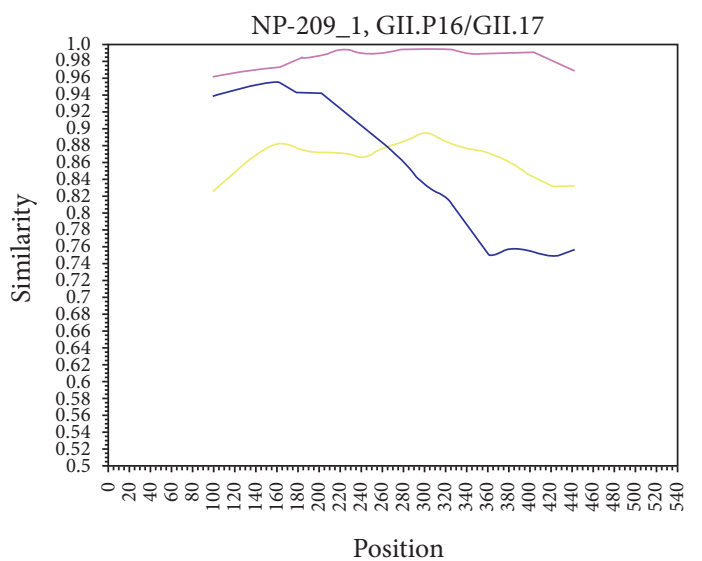

— GII.P16/GII.16_GQ856476_— GII.P16/GII.17_AY502009 GII.P17/GII.17_AB983218

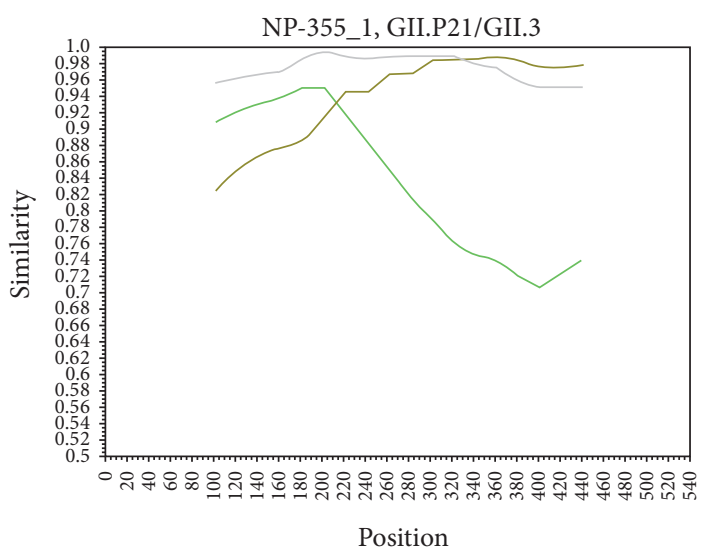

— GII.P3/GII.3_AB112332 — GII.P21/GII.3_KM198586

Window: 200 bp, step: 20 bp, GapStrip: On, Kimura (2-parameter), T/t: 2.0

FIGURE 3: Representative plots of Simplot analysis of each norovirus recombinant. The $y$-axis indicates a degree of similarity between query recombinant and recombinant and two nonrecombinant parental norovirus strains. Sample name and recombination genotype are located on top of each plot. Samples names are indicated as NP \#\#\#-\# for case and NPC \#\#\#-\# for control. \# after the sample name indicates colony number of clone for that sample. Nonrecombinant parental strains used are GII.P3/GII.3-AB112332, GII.P6/GII.6-AB039779, GII.P7/GII.7-AF414409, GII.P12/GII.12-AB525813, GII.P13/GII.13-EU921354, GII.14-AY130761, GII.P16/GII.16-GQ856476, GII.P17/GII,17AB983218, GII.P20/GII.20-EU275779, and GII.P21/GII.21-EU019230. Recombinant parental strains used are GII.P7/GII.6-AB504694, GII.P7/GII.14_EF670650, GII.P7/GII.P20-AB542917, GII.P12/GII.13-AB354294, GII.P16/GII.17-AY502009, and GII.P21/GII.3-KM198586. 
worldwide [7, 37, 38]. Mixed norovirus infections were also detected in 4 cases which might serve as a potential source of inter- and intragenotype norovirus recombination.

Recombination between the RNA-dependent RNA polymerase and the capsid genes has been reported worldwide in recent years [39]. The Norovirus Genotyping Tool and phylogenetic tree analysis of the Cambodian norovirus sequences led to the identification of 7 recombinants with more than $95 \%$ sequence identity to published sequences of recombinant variants (Figure 3 and Table 2). Simplot program was used to confirm 5 recombination genotypes and identified recombination breakpoints at the ORF1/2 junction which correspond to previously reported breakpoints (49815117 nucleotides of Lordsdale virus genome) [27, 40]. Recombination breakpoint could not be confirmed for GI.Pc/GI.5 and GII.P7/GII.14 as nonrecombinant parental strains are not available [3].

All of the recombinants identified in this study have been reported previously from various geographical locations from samples that were collected prior to, after, or at the same time period as this study. GI.Pc/GI.5 and GII.P21/GII.3 were reported earlier than the rest of recombinants in this study with GII.P21/GII.3 being the most widely detected recombinant $[9,21,41]$. The rest of recombinants identified in this study match recombinants reported in 2008 or later suggesting that recombinants identified in this study existed before. However, due to geographical distances, it is likely that norovirus recombinants identified in this study occurred as a separate event and may not be directly related to reported recombinants. Approximately $37 \%$ of norovirus isolates (16/43) in this study were recombinants which suggest that viral recombination has an important role in norovirus success in an evasion of host immune responses as supported by an increase in reports of novel recombinant strains $[9,39]$.

Despite advances in understanding norovirus biology, no norovirus virulence determinants have been identified and there is currently no efficient way to predict which strains will become dominant. Sequence and biochemical studies suggest that norovirus employs at least two mechanisms to persist in the population: antigenic drift and recombination $[7,42]$. Understanding how norovirus evolves and adapts to immunological pressure is critical for the development of an effective vaccine and antiviral therapy.

\section{Conclusions}

This study reports on molecular epidemiology of norovirus circulating in young children in Phnom Penh, Cambodia, form 2004 to 2006. The existence of several GII.4 variants and recombinant strains in Cambodia suggests the need for a continued surveillance system that includes molecular aspects to provide a better epidemiological understanding for the development of vaccines against norovirus.

\section{Disclosure}

The views expressed in this article are those of the authors and do not reflect the official policy of the Department of the Army, Department of Defense, or the US Government.
Tradenames are used for identification purposes only and do not imply endorsement.

\section{Competing Interests}

The authors do not have an association that might pose competing interests.

\section{Acknowledgments}

The authors acknowledge grant from Armed Forces Health Surveillance Center, Division of Global Emerging Infections Surveillance and Response System (AFHSC-GEIS), Silver Spring, MD, USA. The authors thank the staff members of the National Pediatric Hospital for their assistance in the enrollment and laboratory work and staff members of the Department of Enteric Diseases, AFRIMS, for their support in laboratory assays and data management.

\section{References}

[1] M. M. Patel, M.-A. Widdowson, R. I. Glass, K. Akazawa, J. Vinjé, and U. D. Parashar, "Systematic literature review of role of noroviruses in sporadic gastroenteritis," Emerging Infectious Diseases, vol. 14, no. 8, pp. 1224-1231, 2008.

[2] P. J. Glass, L. J. White, J. M. Ball, I. Leparc-Goffart, M. E. Hardy, and M. K. Estes, "Norwalk virus open reading frame 3 encodes a minor structural protein," Journal of Virology, vol. 74, no. 14, pp. 6581-6591, 2000.

[3] J. Vinjé, "Advances in laboratory methods for detection and typing of norovirus," Journal of Clinical Microbiology, vol. 53, no. 2, pp. 373-381, 2015.

[4] A. Kroneman, H. Vennema, K. Deforche et al., "An automated genotyping tool for enteroviruses and noroviruses," Journal of Clinical Virology, vol. 51, no. 2, pp. 121-125, 2011.

[5] J. J. Siebenga, H. Vennema, D.-P. Zheng et al., "Norovirus illness is a global problem: emergence and spread of norovirus gii. 4 variants, 2001-2007,' Journal of Infectious Diseases, vol. 200, no. 5, pp. 802-812, 2009.

[6] M. Okada, T. Tanaka, M. Oseto, N. Takeda, and K. Shinozaki, "Genetic analysis of noroviruses associated with fatalities in healthcare facilities," Archives of Virology, vol. 151, no. 8, pp. 1635-1641, 2006.

[7] L. C. Lindesmith, E. F. Donaldson, A. D. LoBue et al., "Mechanisms of GII.4 norovirus persistence in human populations," PLoS Medicine, vol. 5, no. 2, pp. 0269-0290, 2008.

[8] M. Saito, S. Goel-Apaza, S. Espetia et al., "Multiple norovirus infections in a birth cohort in a peruvian periurban community," Clinical Infectious Diseases, vol. 58, no. 4, pp. 483-491, 2014.

[9] R. A. Bull, M. M. Tanaka, and P. A. White, "Norovirus recombination," Journal of General Virology, vol. 88, no. 12, pp. 33473359, 2007.

[10] S. Fukuda, Y. Sasaki, S. Takao, and M. Seno, "Recombinant norovirus implicated in gastroenteritis outbreaks in Hiroshima Prefecture, Japan," Journal of Medical Virology, vol. 80, no. 5, pp. 921-928, 2008.

[11] I. G. A. Ruether, T. G. Dimitriou, D. Tsakogiannis et al., "Characterization of novel intergenogroup and intergenotype recombinant noroviruses from central Greece," Molecular and Cellular Probes, vol. 28, no. 4, pp. 204-210, 2014. 
[12] C. Y. Meng, B. L. Smith, L. Bodhidatta et al., "Etiology of diarrhea in young children and patterns of antibiotic resistance in Cambodia," Pediatric Infectious Disease Journal, vol. 30, no. 4, pp. 331-335, 2011.

[13] P. V. Tra My, C. Thompson, H. L. Phuc et al., "Endemic norovirus infections in children, Ho Chi Minh City, Vietnam, 2009-2010," Emerging Infectious Diseases, vol. 19, no. 6, pp. 977980, 2013.

[14] L. Bodhidatta, E. Abente, P. Neesanant et al., "Molecular epidemiology and genotype distribution of noroviruses in children in Thailand from 2004 to 2010: a multi-site study," Journal of Medical Virology, vol. 87, no. 4, pp. 664-674, 2015.

[15] P. Neesanant, T. Sirinarumitr, S. Chantakru et al., "Optimization of one-step real-time reverse transcription-polymerase chain reaction assays for norovirus detection and molecular epidemiology of noroviruses in Thailand," Journal of Virological Methods, vol. 194, no. 1-2, pp. 317-325, 2013.

[16] S. Kojima, T. Kageyama, S. Fukushi et al., "Genogroup-specific PCR primers for detection of Norwalk-like viruses," Journal of Virological Methods, vol. 100, no. 1-2, pp. 107-114, 2002.

[17] T. Kageyama, M. Shinohara, K. Uchida et al., "Coexistence of multiple genotypes, including newly identified genotypes, in outbreaks of gastroenteritis due to Norovirus in Japan," Journal of Clinical Microbiology, vol. 42, no. 7, pp. 2988-2995, 2004.

[18] J. D. Thompson, D. G. Higgins, and T. J. Gibson, "CLUSTAL W: improving the sensitivity of progressive multiple sequence alignment through sequence weighting, position-specific gap penalties and weight matrix choice," Nucleic Acids Research, vol. 22, no. 22, pp. 4673-4680, 1994.

[19] K. Tamura, G. Stecher, D. Peterson, A. Filipski, and S. Kumar, "MEGA6: molecular evolutionary genetics analysis version 6.0," Molecular Biology and Evolution, vol. 30, no. 12, pp. 2725-2729, 2013.

[20] K. S. Lole, R. C. Bollinger, R. S. Paranjape et al., "Full-length human immunodeficiency virus type 1 genomes from subtype C- infected seroconverters in India, with evidence of intersubtype recombination," Journal of Virology, vol. 73, no. 1, pp. 152160, 1999.

[21] K. Katayama, H. Shirato-Horikoshi, S. Kojima et al., "Phylogenetic analysis of the complete genome of 18 norwalk-like viruses," Virology, vol. 299, no. 2, pp. 225-239, 2002.

[22] P. Huynen, A. Mauroy, C. Martin et al., "Molecular epidemiology of norovirus infections in symptomatic and asymptomatic children from Bobo Dioulasso, Burkina Faso," Journal of Clinical Virology, vol. 58, no. 3, pp. 515-521, 2013.

[23] M. Jin, H.-P. Xie, Z.-J. Duan et al., "Emergence of the GII4/2006b variant and recombinant noroviruses in China," Journal of Medical Virology, vol. 80, no. 11, pp. 1997-2004, 2008.

[24] T. M. Fumian, G. C. Aragão, J. D. P. Mascarenhas et al., "Detection of a novel recombinant strain of norovirus in an Africandescendant community from the Amazon region of Brazil in 2008," Archives of Virology, vol. 157, no. 12, pp. 2389-2392, 2012.

[25] Y.-J. Won, J.-W. Park, S.-H. Han et al., "Full-genomic analysis of a human norovirus recombinant GII.12/13 novel strain isolated from south korea," PLoS ONE, vol. 8, no. 12, Article ID e85063, 2013.

[26] J. Mans, T. Y. Murray, and M. B. Taylor, "Novel norovirus recombinants detected in South Africa," Virology Journal, vol.11, article 168, 2014.

[27] R. A. Bull, G. S. Hansman, L. E. Clancy, M. M. Tanaka, W. D. Rawlinson, and P. A. White, "Norovirus recombination in
ORF1/ORF2 overlap," Emerging Infectious Diseases, vol. 11, no. 7, pp. 1079-1085, 2005.

[28] J. Buesa, B. Collado, P. López-Andújar et al., "Molecular epidemiology of caliciviruses causing outbreaks and sporadic cases of acute gastroenteritis in Spain," Journal of Clinical Microbiology, vol. 40, no. 8, pp. 2854-2859, 2002.

[29] B. Nyambat, C. Y. Meng, K. Vansith et al., "Hospital-based surveillance for rotavirus diarrhoea in Phnom Penh, Cambodia, March 2005 through February 2007,' Vaccine, vol. 27, no. 5, pp. F81-F84, 2009.

[30] R. Malasao, N. Maneekarn, P. Khamrin et al., "Genetic diversity of norovirus, sapovirus, and astrovirus isolated from children hospitalized with acute gastroenteritis in Chiang Mai, Thailand," Journal of Medical Virology, vol. 80, no. 10, pp. 1749-1755, 2008.

[31] S. Oum, D. Chandramohan, and S. Cairncross, "Communitybased surveillance: a pilot study from rural Cambodia," Tropical Medicine and International Health, vol. 10, no. 7, pp. 689-697, 2005.

[32] S. M. Ahmed, A. J. Hall, A. E. Robinson et al., "Global prevalence of norovirus in cases of gastroenteritis: a systematic review and meta-analysis," The Lancet Infectious Diseases, vol. 14, no. 8, pp. 725-730, 2014.

[33] L. Sai, J. Sun, L. Shao, S. Chen, H. Liu, and L. Ma, "Epidemiology and clinical features of rotavirus and norovirus infection among children in Ji'nan, China," Virology Journal, vol. 10, article 302, 2013.

[34] B. A. Lopman, T. Trivedi, Y. Vicuña et al., "Norovirus infection and disease in an ecuadorian birth cohort: association of certain norovirus genotypes with host FUT2 secretor status," Journal of Infectious Diseases, vol. 211, no. 11, pp. 1813-1821, 2015.

[35] K. Shioda, A. Kambhampati, A. J. Hall, and B. A. Lopman, "Global age distribution of pediatric norovirus cases," Vaccine, vol. 33, no. 33, pp. 4065-4068, 2015.

[36] A. L. Morrow, G. M. Ruiz-Palacios, X. Jiang, and D. S. Newburg, "Human-milk glycans that inhibit pathogen binding protect breast-feeding infants against infectious diarrhea," The Journal of Nutrition, vol. 135, no. 5, pp. 1304-1307, 2005.

[37] T. N. Hoa Tran, E. Trainor, T. Nakagomi, N. A. Cunliffe, and O. Nakagomi, "Molecular epidemiology of noroviruses associated with acute sporadic gastroenteritis in children: global distribution of genogroups, genotypes and GII.4 variants," Journal of Clinical Virology, vol. 56, no. 3, pp. 185-193, 2013.

[38] R. A. Bull, E. T. V. Tu, C. J. McIver, W. D. Rawlinson, and P. A. White, "Emergence of a new norovirus genotype II.4 variant associated with global outbreaks of gastroenteritis," Journal of Clinical Microbiology, vol. 44, no. 2, pp. 327-333, 2006.

[39] P. A. White, "Evolution of norovirus," Clinical Microbiology and Infection, vol. 20, no. 8, pp. 741-745, 2014.

[40] Á. Fajardo, F. L. Tort, M. Victoria et al., "Phylogenetic analyses of Norovirus strains detected in Uruguay reveal the circulation of the novel GII.P7/GII.6 recombinant variant," Infection, Genetics and Evolution, vol. 28, pp. 328-332, 2014.

[41] P. Chhabra, R. K. Dhongade, V. R. Kalrao, A. R. Bavdekar, and S. D. Chitambar, "Epidemiological, clinical, and molecular features of norovirus infections in western India," Journal of Medical Virology, vol. 81, no. 5, pp. 922-932, 2009.

[42] R. A. Bull and P. A. White, "Mechanisms of GII.4 norovirus evolution," Trends in Microbiology, vol. 19, no. 5, pp. 233-240, 2011. 


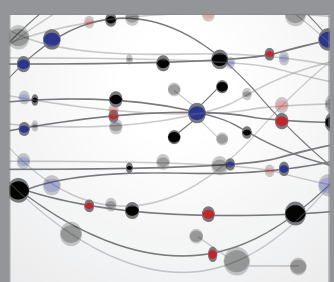

The Scientific World Journal
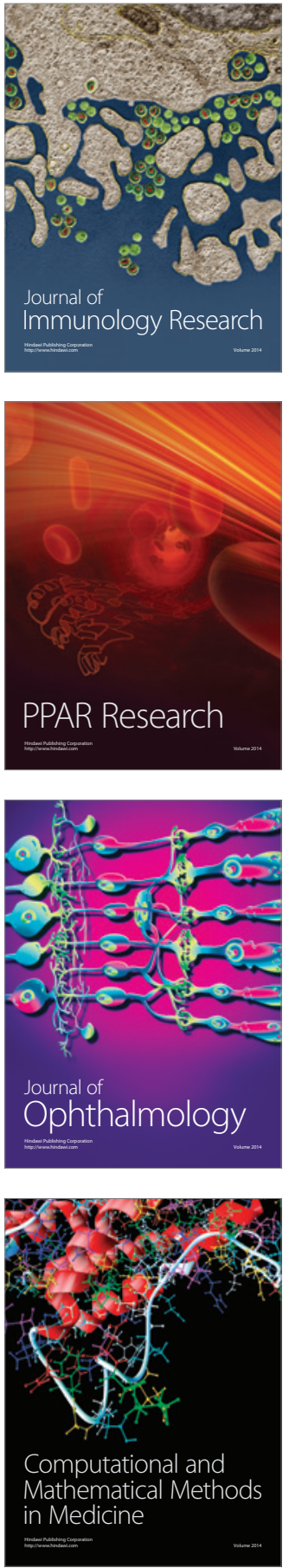

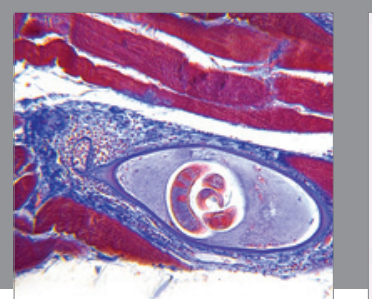

Gastroenterology Research and Practice

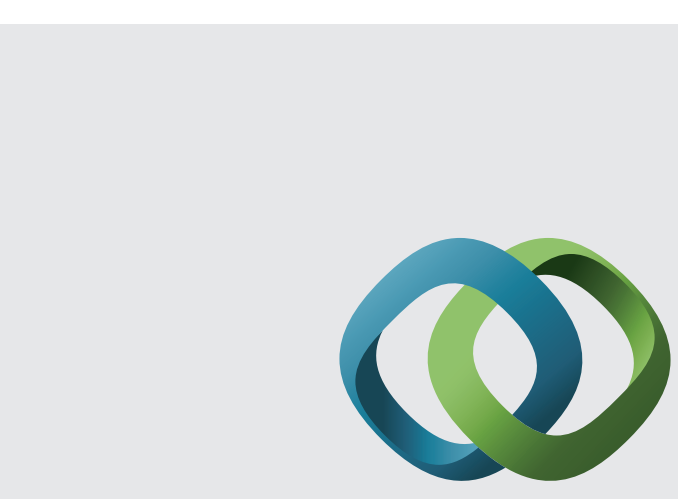

\section{Hindawi}

Submit your manuscripts at

http://www.hindawi.com
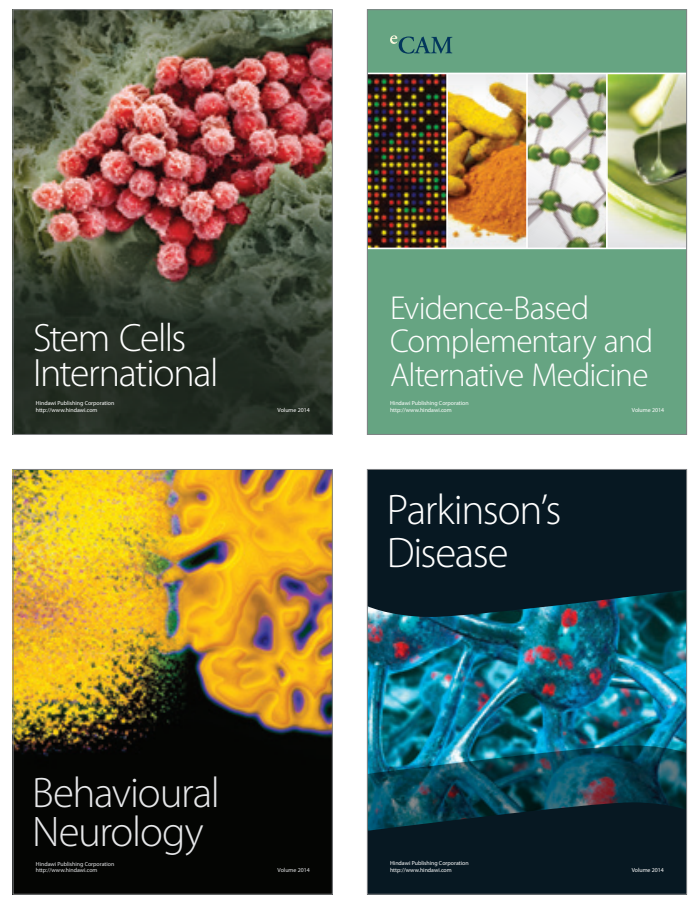
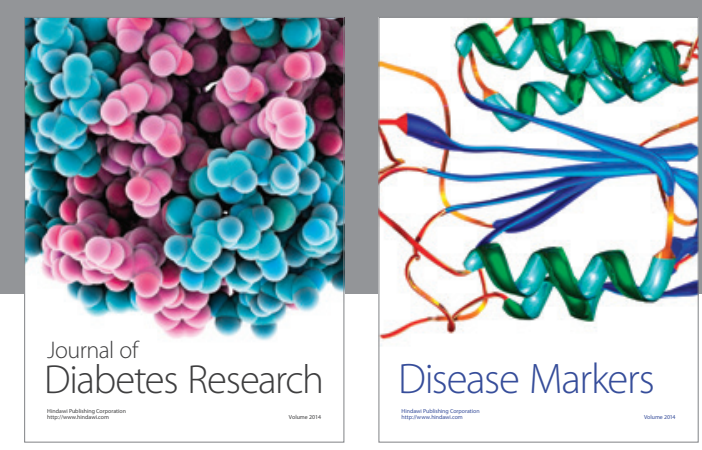

Disease Markers
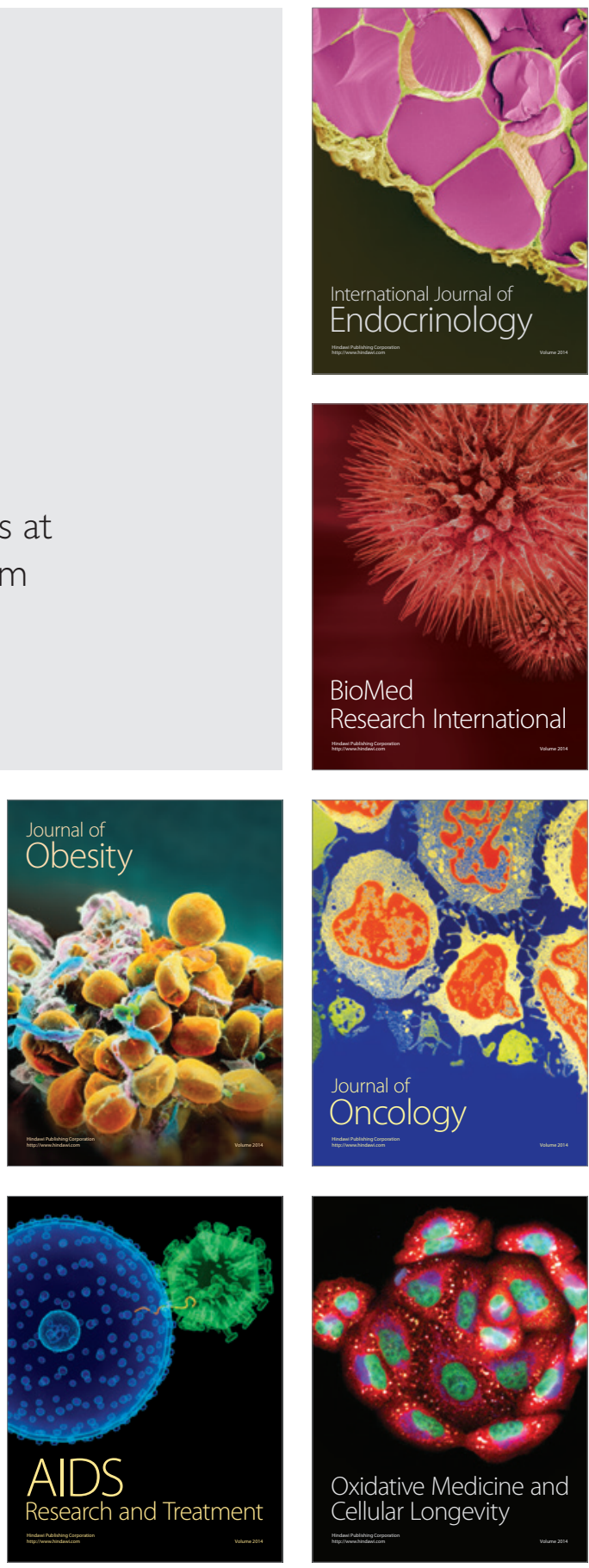